\title{
A simple model for stress-induced anisotropic softening of weak sandstones
}

\author{
M.C. Weng ${ }^{\text {a }}$, F.S. Jeng ${ }^{\text {b,* }}$, Y.M. Hsieh ${ }^{\text {c }}$, T.H. Huang ${ }^{\mathrm{b}}$ \\ ${ }^{a}$ Department of Civil and Environmental Engineering, National University of Kaohsiung, Kaohsiung, Taiwan \\ ${ }^{\mathrm{b}}$ Department of Civil Engineering, National Taiwan University, Taipei, Taiwan \\ ${ }^{\mathrm{c}}$ Department of Construction Engineering, National Taiwan University of Science and Technology, Taipei, Taiwan
}

Received 7 September 2006; received in revised form 23 March 2007; accepted 2 April 2007

Available online 22 May 2007

\begin{abstract}
Weak sandstones possess deformational behavior different from hard rocks; these phenomena include shear dilation and softening of the deformational moduli. It has been found previously that under hydrostatic loading, the bulk modulus increases as confining pressure arises; and that under shear loading, the weak sandstone may transform from its original isotropy to a stress-induced anisotropic material, and the deformational modulus can accordingly be softened as well. These phenomena contribute to the increase of crown settlements during tunnel excavations, and account for several cases of tunnel squeezing. Consequently, a model capable of simulating major deformational characteristics of weak sandstones is needed for engineering purposes. A simple yet innovative constitutive model is accordingly proposed. This proposed model is characterized during the simulation as having: (1) non-linear volumetric deformation under hydrostatic loading; (2) significant shear dilation prior to the failure state; (3) isotropic stiffening of deformational moduli under hydrostatic loading; and (4) anisotropic softening of deformational moduli under shearing condition.

The proposed model was formulated based on the linear elastic model, and it accounts for the variations of moduli $E$ and $G$ through different loading conditions. It was found that the proposed model is able to closely simulate the actual deformational characteristics of weak sandstones. In addition, the proposed model only needs six material parameters, and all these parameters can be easily obtained from experiments. This model was then incorporated into a finite element program and was used to analyze a squeezing tunnel case.
\end{abstract} (C) 2007 Elsevier Ltd. All rights reserved.

Keywords: Weak sandstones; Constitutive model; Shear dilation; Deformational modulus; Softening; Stiffening

\section{Introduction}

Engineering difficulties are often encountered during tunnel constructions in weak rocks, which possess undesirable engineering characteristics such as high porosity, poor cementation, and low strength and stiffness. In the western part of Taiwan, weak sedimentary rocks (including sandstone, shale and mudstone) are often encountered during tunnel constructions, and these materials exhibit relatively low shear strength and stiffness. In several unsuccessful tunneling projects constructed in weak rock strata, ground squeezing and tunnel face instability have been reported [1]. In one particular case where a typical

\footnotetext{
${ }^{*}$ Corresponding author. Tel./fax: + 886223645734.

E-mail address: fsjeng@ntu.edu.tw (F.S. Jeng).
}

weak rock in Taiwan, known as Mushan sandstone, was encountered, several tunnel sections showed a crown settlement ranging from $14-30 \mathrm{~cm}$.

In order to identify the deformational behavior of weak sandstones, several research projects were conducted by the authors. Jeng et al. [2] categorized mechanical properties of many types of sandstones using the petrographic features and categorized the analyzed sandstones into Type $A$ and Type $B$ sandstones. The Type $B$ sandstone, compared to Type $A$, showed lower stiffness and larger reduction in both stiffness and strength during shearing. These characteristics suggested that the Type $B$ sandstone could be a problematic rock type, and tunneling in the Type $B$ sandstone is prone to tunnel squeezing.

For the conventional triaxial compression $(C T C)$ tests, both the confining pressure and shear stresses increase; 
therefore, it is difficult to distinguish the effects of shear and volumetric stresses on the volumetric deformation. As such, triaxial tests with pure shear $(P S)$ stress path (Fig. 1) were performed on weak sandstones to study the effects of volumetric and shear stresses on the volumetric deformation $[3,4]$. Triaxial tests with $P S$ stress path make it possible to separate the effects of shear stresses and volumetric stresses on deformations, and have been adopted in recent years for studying rock mechanics in the three-dimensional stress space. In previous researches, several weak sandstones had been tested. The physical and mechanical properties of specimens were measured according to the methods suggested by the ISRM [3]. The specimen size is $5.5 \mathrm{~cm}$ in diameter and $12.5 \mathrm{~cm}$ in height. The longitudinal and transverse deformation were separately measured by a full Wheatstone bridge consisted of four strain gages, which are capable of measuring strains up to $2 \%$ with an accuracy of $\pm 0.85(\mu \mathrm{m} / \mathrm{m}) /{ }^{\circ} \mathrm{C}$.

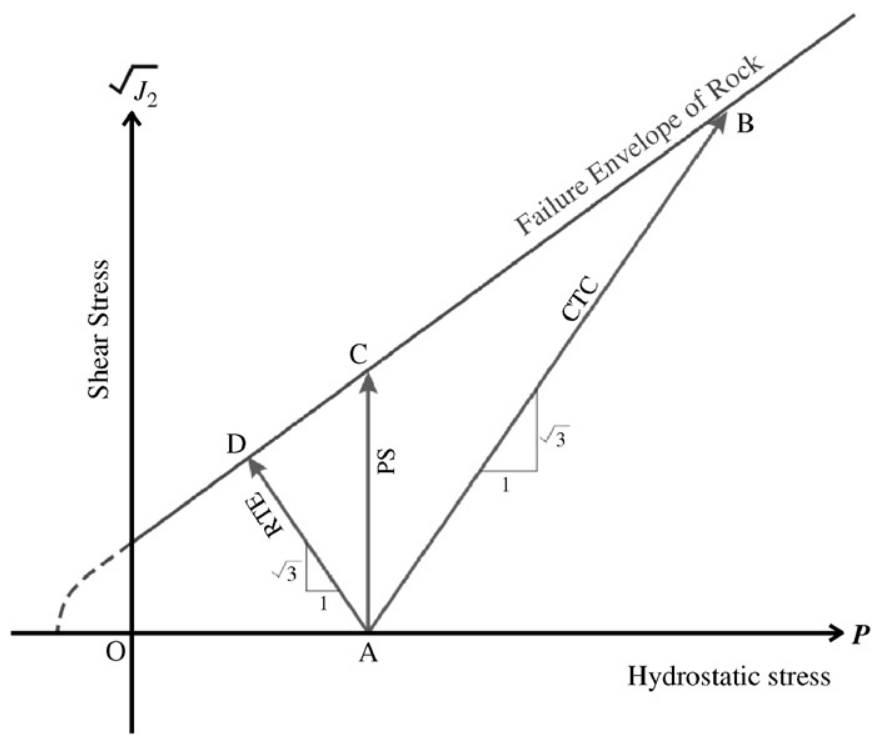

Fig. 1. Schematic illustration of stress paths. $P S=$ pure shear test, $C T C=$ conventional triaxial compression test, $R T E=$ reduced triaxial extension tests.
The specimens were loaded in a triaxial cell, by which the axial pressure and the confining pressure are applied, under room temperature. The triaxial cell is able to sustain a confining pressure up to $175 \mathrm{MPa}$ and the confining pressure was monitored by a pressure transducer with an accuracy of $0.1 \mathrm{MPa}$. The axial load was provided by a servo-controlled high stiffness machine, which has a maximum load and a stiffness of $4448 \mathrm{kN}$ and $13.1 \times 10^{9} \mathrm{~N} / \mathrm{m}$, respectively. The load is applied at a rate of $5 \mathrm{MPa} / \mathrm{min}$. Table 1 summarizes the corresponding testing parameters of all tests referred in this research. Based on the experiment results [4,5], the deformation characteristics of weak sandstones include: (a) non-linear volumetric deformation during hydrostatic compression, and an increase in stiffness with increasing hydrostatic stress (Fig. 2; see also [6]), (b) significant shear dilation and distortion (Figs. 3 and 4) during shearing; and (c) substantial plastic deformation occurs prior to the failure state during shearing.

From micro-mechanical point of view, the plastic behavior is related to the growth of complex micro- and meso-cracks, and accumulation of these cracks and further application of loading will lead to stress-induced anisotropy and softening of rock [7-14]. Sayers et al. [9,10] showed that initially isotropic rocks would exhibit anisotropic elastic behaviors under deviatoric shearing, and the degree of anisotropy would be influenced by the microcrack opening and their orientation. They have also showed the direction of crack propagation is often parallel to the direction of the maximum compressive stress applied. Lockner and Beeler [13] pointed out that when the specimen was subjected to high differential stresses, new micro-crack-induced damage would be initiated, which in turn induced significant anisotropy. They have also found that increasing confining pressure not only increases the deformation moduli but also tends to suppress anisotropy. Olsson [14] indicated that changes in the incremental shear moduli reflect the development of deformation-induced, anisotropic damage. He measured the incremental shear moduli in two directions, and found that in the initial loading range the moduli were equal, but then the moduli decreased in different rates with increasing loadings.

Table 1

Testing program of weak sandstone

\begin{tabular}{llll}
\hline Type of tests & Test code & Confining pressure (MPa) & No. of tests \\
\hline Pure shear stress path test & MS20 & 20 & 3 \\
& MS40 & 40 & $3+1^{\mathrm{a}}$ \\
& MS60 & 60 & 80 \\
1 & MS80 & 2 & 10 \\
Conventional triaxial test $(C T C)$ & MSC02 & 18 & 1 \\
& MSC10 & 40 & 1 \\
RSC18 & MSC40 & 40 & 2 \\
\hline
\end{tabular}

${ }^{\mathrm{a}}$ Cyclic loading/unloading was performed. 


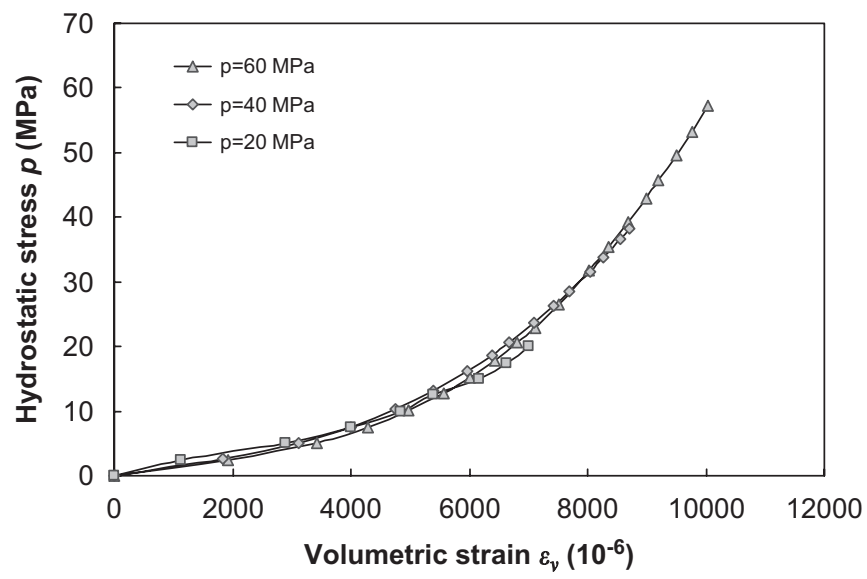

Fig. 2. Typical deformation curves of volumetric strain induced by volumetric stress (Path OA in Fig. 1) obtained from weak sandstones.

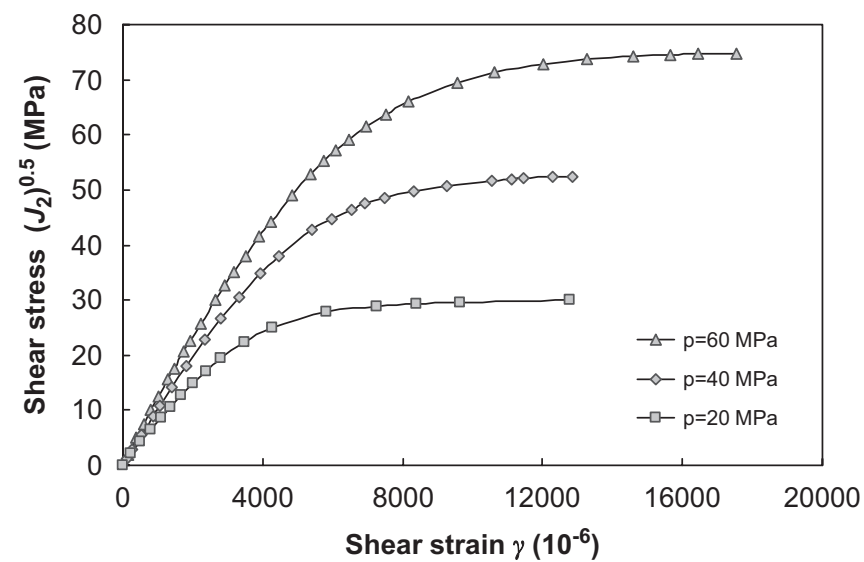

Fig. 3. Typical deformation curves of shear strain induced by shear stress (Paths AC in Fig. 1).

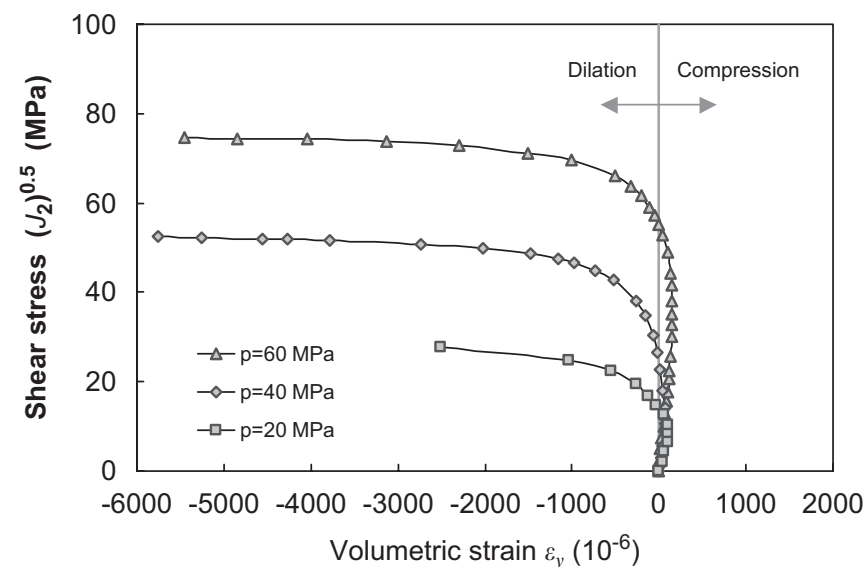

Fig. 4. Typical deformation curves of volumetric strain induced by shear stress (Path AC in Fig. 1).

In addition to understanding deformation behaviors, including shear dilation and stress-induced anisotropy of weak rocks, the assessment of the stress condition and displacements associated with these deformation characteristics is important in practical civil engineering or in rock engineering. Conventionally, rocks are often considered as linear-elastic, followed by plastic behaviors before and after the stress state reaches the yielding condition of the material, respectively. This model probably works well for hard rocks, in which the shear dilation or the stress-induced anisotropy are not significant, but performs unsatisfactorily for soft rocks in the following two regards. Firstly, as shear dilation and stress-induced anisotropy exist, they need to be incorporated into a suitable constitutive model, especially for tunnel constructions in the squeezing ground, in which the volumetric deformation is significant. Furthermore, the softening of the stiffness modulus occurs even in the socalled elastic stage, and this nonlinear elasticity should be considered in establishing a constitutive model for soft rocks.

Conventional elasto-plastic models may require quite a few material parameters on the one hand, or may still have limited capability of describing the aforementioned deformational characteristics of soft rocks on the other hand. Therefore, a simple yet effective constitutive model, which is characterized by only a few material parameters for soft rocks, is needed for engineering design and analysis. Accordingly, an innovative constitutive model accounting for stress-induced anisotropy and softening of weak rocks is proposed in this study.

The proposed model was designated to be capable of showing the following characteristics:

1. The model gives reasonable predictions on apparent shear dilation, including the stress-induced anisotropic softening;

2. The relatively simple model can be readily applicable to engineering practice by requiring a simple and straightforward parameter determination procedure;

3. Relatively fewer material parameters, compared to complicated elasto-plastic models, are needed in the proposed model; and,

4. The major deformational behaviors of weak rocks can be well described by the proposed model.

\section{Model formulation}

\subsection{Elastic modeling for stress-induced anisotropic softening}

For isotropic linear elastic material, the matrix form of the incremental stress-strain relations in the principal stress space can be expressed as

$$
\left\{\begin{array}{l}
\Delta \varepsilon_{1} \\
\Delta \varepsilon_{2} \\
\Delta \varepsilon_{3}
\end{array}\right\}=\frac{1}{E}\left[\begin{array}{ccc}
1 & -\frac{E-2 G}{2 G} & -\frac{E-2 G}{2 G} \\
-\frac{E-2 G}{2 G} & 1 & -\frac{E-2 G}{2 G} \\
-\frac{E-2 G}{2 G} & -\frac{E-2 G}{2 G} & 1
\end{array}\right]\left\{\begin{array}{l}
\Delta \sigma_{1} \\
\Delta \sigma_{2} \\
\Delta \sigma_{3}
\end{array}\right\},
$$

where $E$ and $G$ are tangent Young's modulus and shear modulus; $\Delta \sigma_{1}, \Delta \sigma_{2}$ and $\Delta \sigma_{3}$ are the stress increments along 
the three directions of principal stresses; and $\Delta \varepsilon_{1}, \Delta \varepsilon_{2}$ and $\Delta \varepsilon_{3}$ are the corresponding strain increments.

The laboratorial experiments, as shown in Fig. 2, found that the bulk modulus $K$ can be related to the hydrostatic stress as

$K=a \frac{I_{1}}{3}+b$,

where $I_{1}$ is the first stress invariant; $a$ and $b$ are the material parameters. Parameter $b$ is the bulk modulus of the material at zero confinement. Parameter $a$ determines the rate of increase of bulk modulus with increasing confining pressure. Based on same observation regarding the nonlinear behavior, a similar formula also has been proposed by Zimmerman [6].

A constitutive model for weak sandstones can be developed to describe the softening and the stress-induced anisotropy by modifying Eq. (1). The concept of the proposed model is described as follows: the weak sandstone subjected to hydrostatic stress condition can be viewed as an isotropic material with deformation modulus that increases or stiffens when confining pressure increases. As such, the material parameters $a$ and $b$ in Eq. (2) can be related to the initial shear modulus (i.e., maximum shear modulus) based on the isotropic elasticity theory as

$G_{\max }=\frac{3 K(1-2 v)}{2(1+v)}=\frac{3(1-2 v)}{2(1+v)}\left(a \frac{I_{1}}{3}+b\right)$,

where $v$ is the Poisson's ratio in Eq. (3).

When the material is subjected to shear loadings, the shearing may cause both softening of deformation moduli and stress-induced anisotropy. Like soil materials, the stress-strain behavior of weak sandstones is highly nonlinear at all phases of shear loading with a maximum shear modulus $G_{\max }$ at initial, small strain. Therefore, the modulus degradation (softening) can be expressed as a function (Eq. (4)) of mobilized stress level, or $G / G_{\max }$ vs. shear stress. Similar relationships have also been proposed for other geomaterials. [15-17].

$G=G_{\max }\left[1-\left(\frac{\sqrt{J_{2}}}{\sqrt{J_{2, f}}}\right)^{2}\right]$,

where the shear strength $\sqrt{J_{2, f}}$ can be calculated by assuming a linear yield criteria which has the form as: $\sqrt{J_{2, f}}=\alpha I_{1}+k$; and $\sqrt{J_{2}}$ is the second deviatoric stress invariant.

Based on observations from experiments [2,13,14], it was found that the application of shear stress will induce anisotropy and two assumptions can accordingly be made for the proposed constitutive model: the direction of "anisotropic" softening is assumed to be coincident with the axis of the major principal stress based on experimental observations; and the shear modulus degradations parallel to the major principal stress axis can be described by Eq. (4). Furthermore, the shear modulus degradation normal to the major principal stress direction can be described using a similar relationship as Eq. (4) by introducing an anisotropic parameter $e$ ranging from 0 to 1 :

$G^{\prime}=G_{\max }\left[1-\left(e \frac{\sqrt{J_{2}}}{\sqrt{J_{2, f}}}\right)^{2}\right]$.

Therefore, for materials like weak sandstones, the matrix of the stress-strain relation in the principal stress coordinate can be expressed as

$$
\left\{\begin{array}{l}
\Delta \varepsilon_{1} \\
\Delta \varepsilon_{2} \\
\Delta \varepsilon_{3}
\end{array}\right\}=\frac{1}{E}\left[\begin{array}{ccc}
1 & -\frac{E-2 G}{2 G} & -\frac{E-2 G}{2 G} \\
-\frac{E-2 G}{2 G} & 1 & -\frac{E-2 G^{\prime}}{2 G^{\prime}} \\
-\frac{E-2 G}{2 G} & -\frac{E-2 G^{\prime}}{2 G^{\prime}} & 1
\end{array}\right]\left\{\begin{array}{c}
\Delta \sigma_{1} \\
\Delta \sigma_{2} \\
\Delta \sigma_{3}
\end{array}\right\} .
$$

If $G=G^{\prime}$, the matrix is identical to the matrix of an isotropic material and the material is still an isotropic material. As will be shown later, $G^{\prime}$ can differ from $G$ under certain loading conditions and the matrix has a form different from the matrix of isotropic materials. Under such circumstance, the material is referred as a stressinduced anisotropic material.

Remarkably, if $G \neq G^{\prime}$, the material is anisotropic and the increments $\left(\Delta \sigma_{1}, \Delta \sigma_{2}, \Delta \sigma_{3}, \Delta \varepsilon_{1}, \Delta \varepsilon_{2}\right.$ and $\left.\Delta \varepsilon_{3}\right)$ in Eqs. (1) and (6) should be associated with the major, intermediate and minor stress or strain increments, respectively.

The deformation modulus $E$ can be expressed in terms of $G$ and $v$ as

$$
\begin{aligned}
E & =2(1+v) G \\
& =2(1+v) G_{\max }\left(1-\left(\frac{\sqrt{J_{2}}}{\sqrt{J_{2, f}}}\right)^{2}\right) .
\end{aligned}
$$

From Eqs. (4),(5) and (7), the variations of moduli $E, G$ and $G^{\prime}$ in the stress space are plotted in Fig. 5, which clearly depicts how Young's modulus and shear modulus vary with different combinations of hydrostatic stress and shear stress.

Based on Eq. (6) and the elasticity theory, the volumetric strain increment can be obtained as Eq. (8), and the volumetric strain can be further separated into two components: volumetric deformation due to hydrostatic stress $\left(\Delta \varepsilon_{v}\right)_{p}$, and volumetric deformation due to shear stress $\left(\Delta \varepsilon_{v}\right)_{s}$ as the following expressions:

$$
\begin{gathered}
\Delta \varepsilon_{v}=\left(\Delta \varepsilon_{v}\right)_{p}+\left(\Delta \varepsilon_{v}\right)_{s}=\frac{1}{3 E}\left(9-\frac{2 E}{G}-\frac{E}{G^{\prime}}\right) \Delta I_{1} \\
+\frac{\left(G-G^{\prime}\right)}{3 G \cdot G^{\prime}} \sqrt{\frac{2}{3} \Delta J_{2}}, \\
\left(\Delta \varepsilon_{v}\right)_{p}=\frac{1}{3 E}\left(9-\frac{2 E}{G}-\frac{E}{G^{\prime}}\right) \Delta I_{1},
\end{gathered}
$$


a

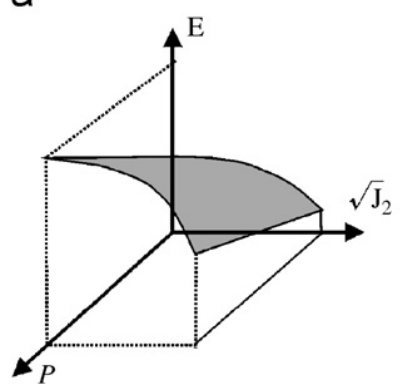

b

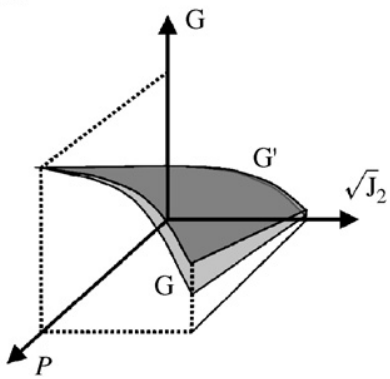

Fig. 5. Schematic illustration of the variation of the deformation modulus $E, G$ and $G^{\prime}$ in the hydrostatic stress and shear stress space.

$\left(\Delta \varepsilon_{v}\right)_{s}=\frac{\left(G-G^{\prime}\right)}{3 G \cdot G^{\prime}} \sqrt{\frac{2}{3} \Delta J_{2}}$,

Eq. (10) shows two interesting features of the proposed model: (1) Shearing induces volumetric deformations, and this phenomena cannot be described using isotropic linear elastic models; and (2) Shearing can produce both compressive and dilative volumetric strains. If $G$ is greater than $G^{\prime}$, the volumetric strain increment $\left(\Delta \varepsilon_{v}\right)_{s}$ will be compressive; if $G$ is smaller than $G^{\prime}$, the $\left(\Delta \varepsilon_{v}\right)_{s}$ will be dilative. As such, this proposed model has the ability of properly describing the shear-induced deformation observed in lab experiments.

\subsection{Determination of material parameters}

The elastic moduli $E, G$ and $G^{\prime}$ of the proposed elastic model can be deduced from six material parameters $a, b, e$, $v, \alpha$ and $k$. Parameters $a$ and $b$ determine the volumetric behavior of the material under isotropic loading, as shown in Eq. (2). Parameter $e$ dictates the stress-induced anisotropy, or different degrees of shear modulus degradations in different principal directions, as in Eqs. (4) and (5). Parameter $v$ is the Poisson's ratio used in Eqs. (3) and (7). Parameters $\alpha$ and $k$ are parameters related to the shear strength of the material, and they are used in the proposed model to define softening behavior, as in Eqs. (4) and (5).

This section demonstrates how these six parameters can be determined during laboratory experiments by using an example of the Mushan sandstone, a weak rock commonly seen in mountain areas in Taiwan. The Mushan sandstone, which has caused squeezing ground hazards for tunnels in Taiwan, has a porosity of $14.1 \%$, dry density of $2.3 \mathrm{~g} / \mathrm{cm}^{3}$, and saturated water content of $5.8 \%$. The average uniaxial compressive strength is $37.1 \mathrm{MPa}$ in dry condition and $28.9 \mathrm{MPa}$ in saturated condition. Based on the petrographic analyses, the percentages of grains, matrix, and voids are $67.5 \%, 18.5 \%$ and $14.1 \%$. The average particle diameter is $0.72 \mathrm{~mm}$. Mineralogically, Mushan sandstone consists of $90.7 \%$ of quartz, $9.0 \%$ of rock fragments, and thus is classified as lithic graywacke [2,4].

A total of six material parameters are required for the proposed constitutive model. As such, two laboratory experiments are needed: (1) uniaxial compression tests, and
(2) triaxial compression tests with PS stress path. The data needed to be measured from the experiments include: the axial strain, the lateral strain, uniaxial compressive strength and the shear strength under different hydrostatic stress.

Poisson's ratio $v$ can be obtained from uniaxial compression tests at the initial loading stage. From hydrostatic loading stage of triaxial compression tests, as shown in Fig. 6, material parameters $a$ and $b$ can be determined by curve fitting Eq. (2) with experimental curves. It can be seen from Fig. 6 that the bulk modulus varies almost linearly with the confining pressure, and therefore the proposed linear relationship (Eq. (2)) is applicable and can be accordingly determined by fitting the experimental curve shown in Fig. 6.

During shear loading stage of triaxial compression tests, parameters $\alpha, k$, and $e$ can be determined. In this proposal model, a yield criterion similar to the Drucker-Prager model is assumed and used to determine the shear strength of the material. These three parameters in turn will affect the shear modulus degradation during softening. The parameters $\alpha$ and $k$ are the friction angle and the cohesion intercept of the yield criterion, as shown in Fig. 7. The parameter $e$, which ranges from 0 to 1 , reflects the degree of shear-induced anisotropy. A decreasing $e$ increases the difference between shear moduli $G$ and $G^{\prime}$ as well as it results in more shear dilation, which is illustrated in Fig. 8(b). The parameter $e$ can be back calculated from the volumetric deformation under shear loading.

\section{Results of simulations using the proposed model}

The performance of the proposed model is demonstrated by comparing the laboratory results with the model simulations. The versatility of the proposed constitutive model is then tested by using the same set of fitted parameters to predict material responses under different testing conditions or stress paths, and these predictions are compared with laboratory experiment data.

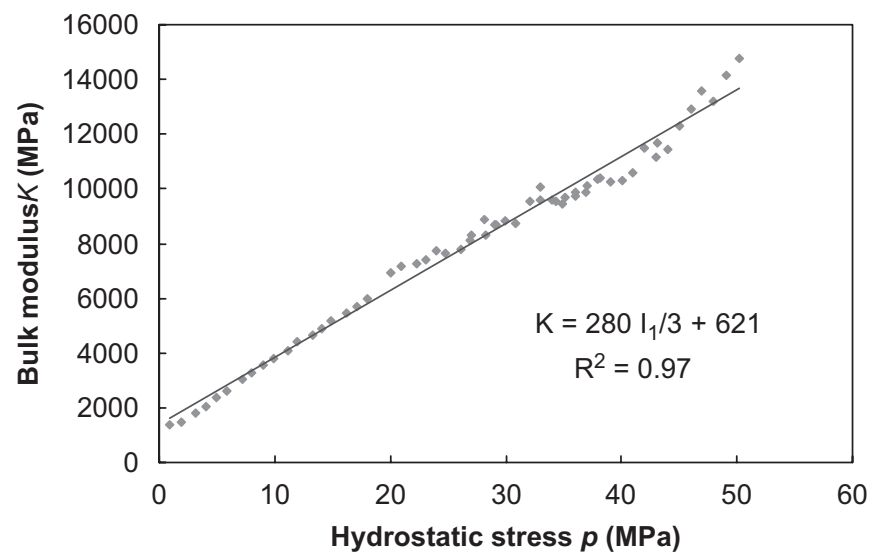

Fig. 6. Variation of tangent bulk modulus $K$ during hydrostatic compression state of loading. Three test results are shown in the figure. By fitting the experiment results, the material parameters $a$ and $b$ are accordingly determined, as listed in Table 2 . 


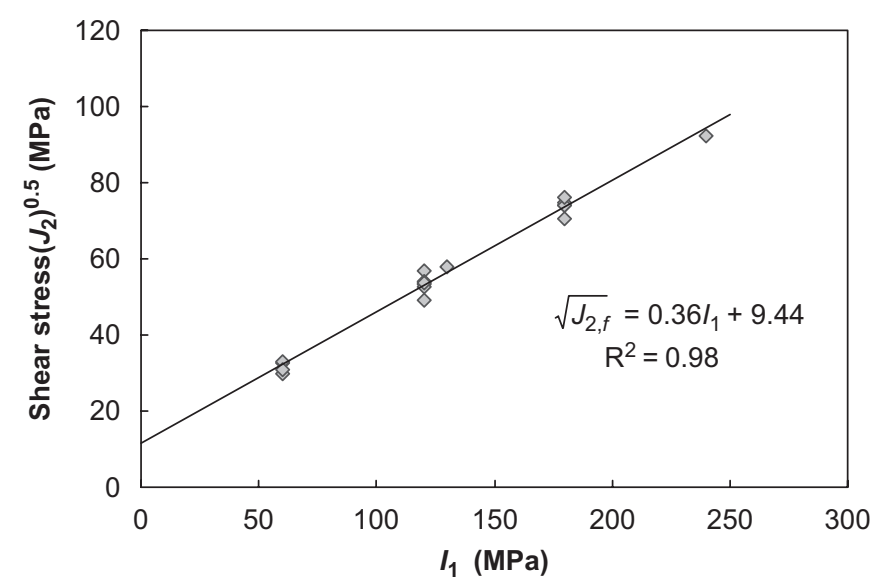

Fig. 7. The yield criterion of the studied weak sandstone.

\subsection{Simulations of experimental results}

One PS experiment conducted using the Mushan sandstone in the laboratory is simulated by the proposed model with fitted parameters for this experiment listed in Table 2, and the measured and simulated deformation stress-strain curves obtained from $P S$ experiments are shown in Fig. 8. At the hydrostatic loading stage of the experiment, it is seen, as in Fig. 8(a), that the measured nonlinear volumetric behavior can be well simulated by the proposed model. As the experiment continues at the shear loading stage, as shown in Fig. 8(b), the material shows significant shear dilation, which is also well simulated by the model simulation. As a result, the proposed model is capable of modeling deformational behavior of weak sandstones under both hydrostatic loading and shear loading conditions.

Deducing from the experimental results, the variations of moduli $E, G$ and $G^{\prime}$ for the two loading stages are plotted in Fig. 8(c). At first, all moduli increase linearly, as described by Eqs. (2) and (3), under hydrostatic loading. Afterwards, as shear stress is applied, all moduli degrade exponentially according to Eqs. (4) and (5), and $G$ starts to differ from $G^{\prime}$ upon increasing shearing, as shown in Fig. 8(c). Therefore, the studied sandstone deforms isotropically and anisotropically upon hydrostatic and $P S$, respectively. Based on Eq. (7), the proposed model is able to simulate not only the stress-induced anisotropy of a material, which is initially isotropic, but also the softening of the deformation. Overall, the proposed model is capable of simulating the deformation behavior of weak sandstones induced by both the hydrostatic and pure shearing loadings.

\subsection{Versatility of the proposed model for different stress paths}

In order to evaluate the predictive capability of the proposed model, the same set of material parameters listed

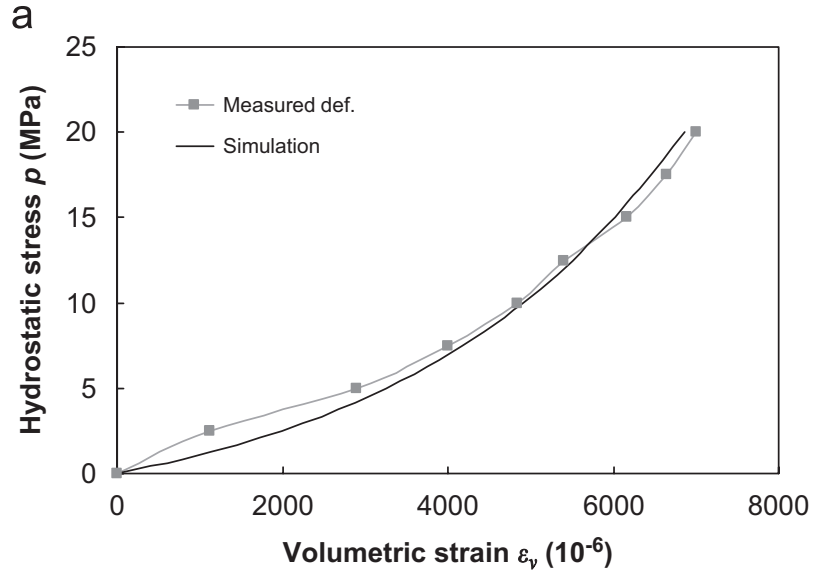

b
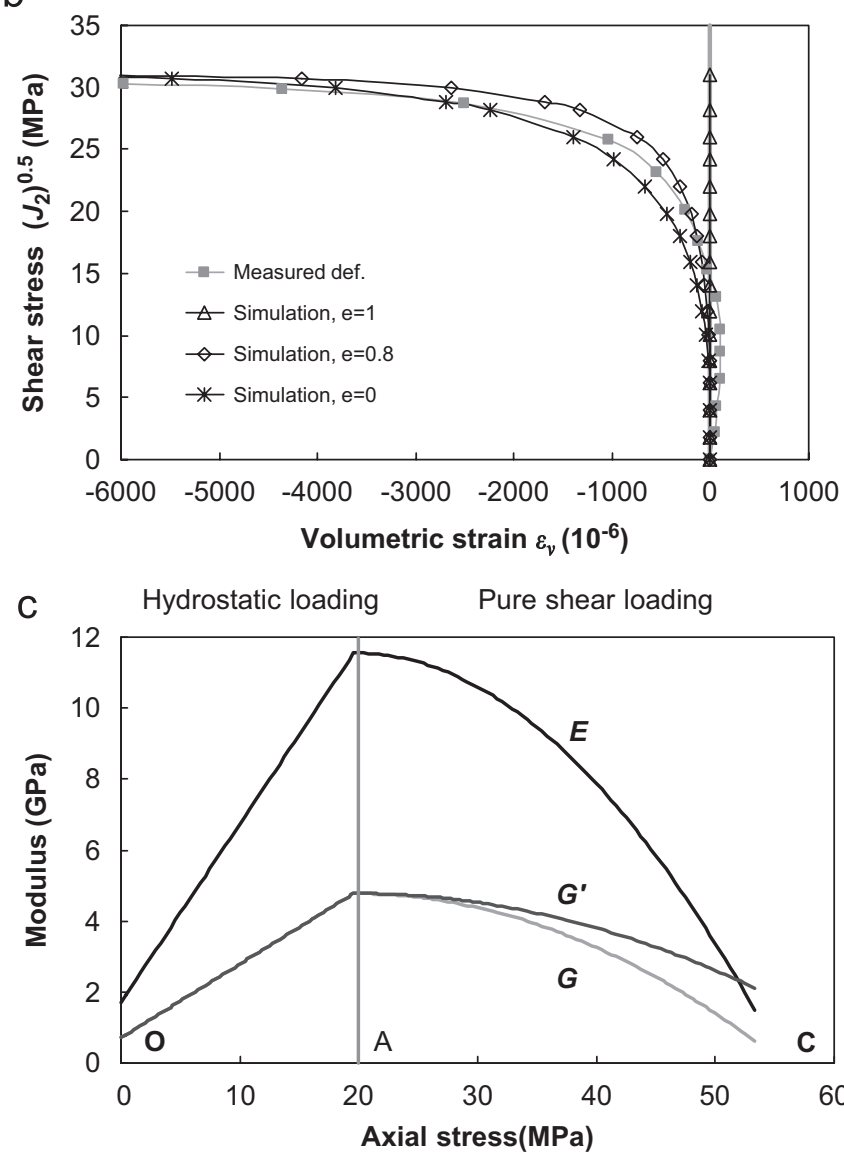

Fig. 8. Comparison of the deformation predicted by the proposed model and by the $P S$ experiments: (a) Variation of volumetric strain during hydrostatic loading; (b) shear dilation induced by pure shear loading. The variation of the deformational curves upon various $e$ is also plotted. Accordingly, the material parameter $e$ is determined to be 0.8 by bestfitting: (c) variation of deformational moduli deduced from experiments.

in Table 2 is used for the proposed model to predict material responses under various confining stresses and shear stress paths, and these predictions are then compared to laboratory measures.

Fig. 9 shows the $P S$ results of both model predictions and experimental results under three different hydrostatic 
Table 2

Material coefficients of Mushan Sandstone for the proposed constitutive model

\begin{tabular}{lll}
\hline Coefficients & Remark & Value \\
\hline$a$ & Material parameter & 280 \\
$b$ & Material parameter & $621 \mathrm{MPa}$ \\
$v$ & Poisson's ratio & 0.21 \\
$e$ & Material parameter & 0.8 \\
$\alpha$ & Slope of failure criteria & 0.36 \\
$k$ & Interception of failure criteria & $9.44 \mathrm{MPa}$ \\
\hline
\end{tabular}

a

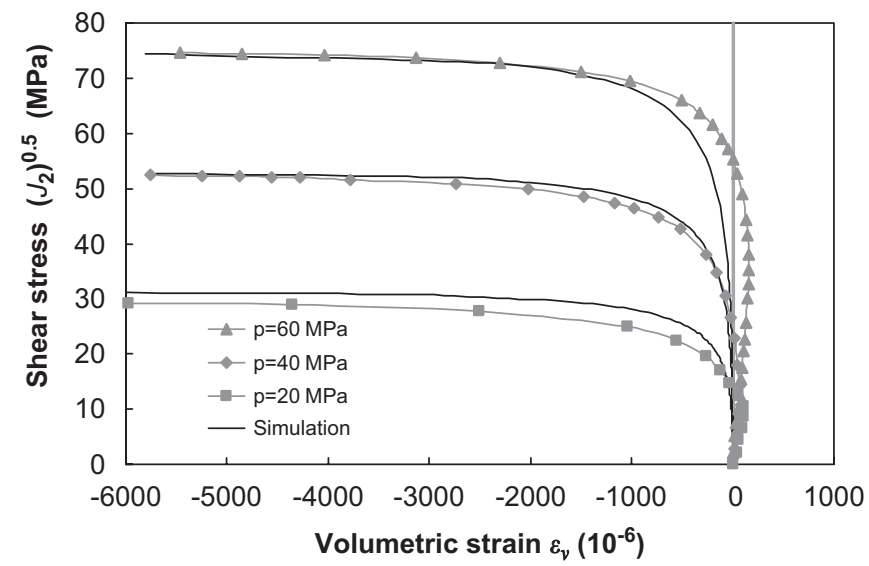

b

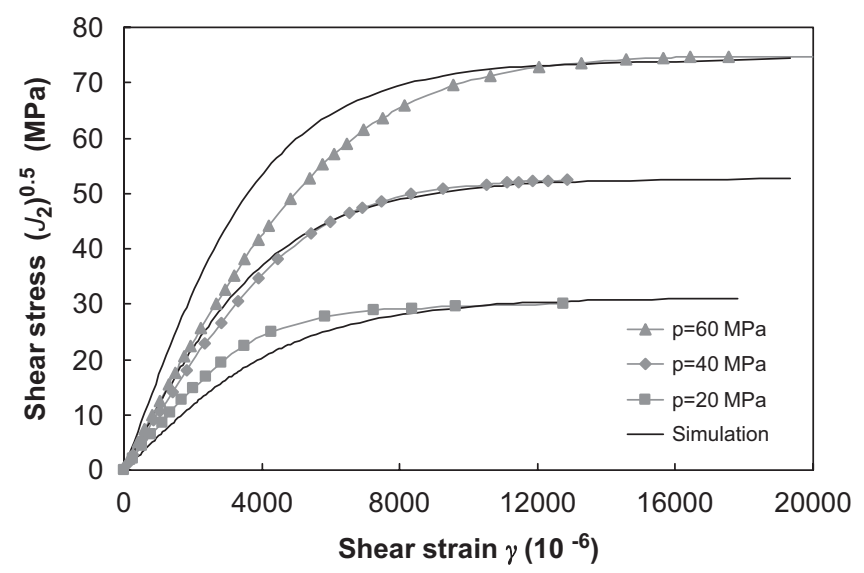

Fig. 9. Simulation of shear and volumetric strains induced by shear stress under different hydrostatic pressures ( $P S$ tests). From Figs. 9-11, the material parameters were obtained from $P S$ test under $p=20 \mathrm{MPa}$.

stresses, $p=20,40$ and $60 \mathrm{MPa}$. It can be seen in Fig. 9 that the proposed model gives reasonable predictions, which compares well to the measured deformation in the aspects of volumetric deformation (Fig. 9(a)) and shear deformation (Fig. 9(b)). It should be emphasized that the parameters are obtained by fitting the experimental results tested under $p=20 \mathrm{MPa}$, thus the simulations for $p=40$ and $60 \mathrm{MPa}$ are actual predictions rather than results obtained from curve fittings.

To further evaluate the versatility of the proposed model, different stress paths (the CTC tests) are simulated

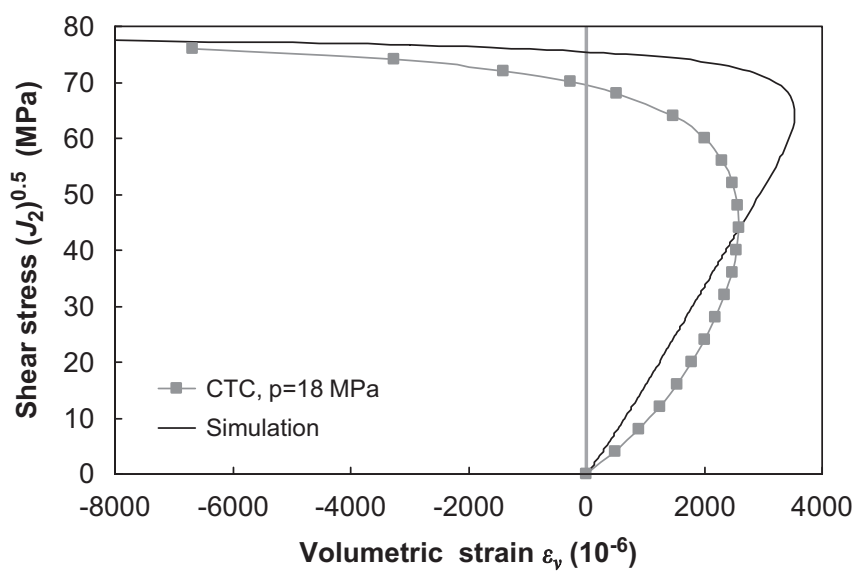

Fig. 10. Simulation of volumetric strains induced by shear stress for $C T C$ tests.

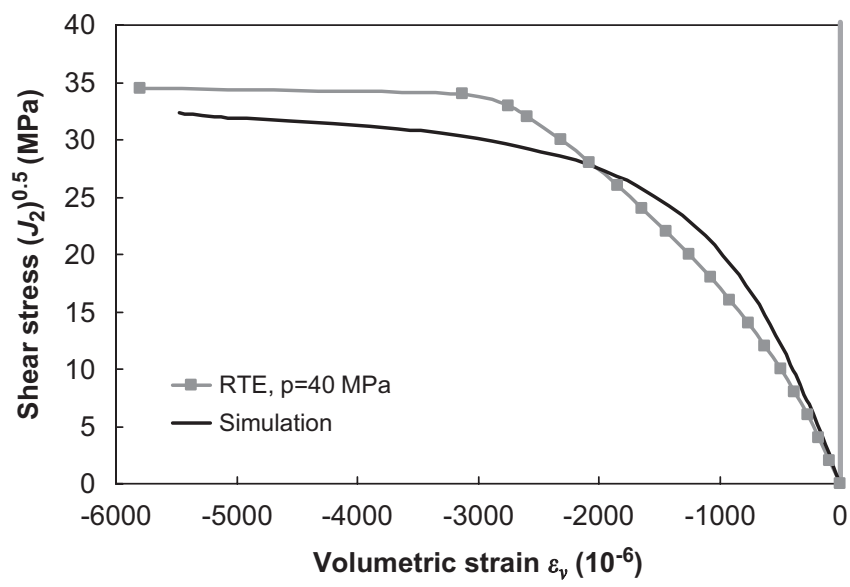

Fig. 11. Simulation of volumetric strains induced by shear stress for RTE tests.

by the proposed model using parameters in Table 2, which was obtained from the $P S$ experiment under $p=20 \mathrm{MPa}$. It can be seen in Fig. 10 that the model prediction shows shear contraction followed by shear dilation volumetric behavior, which is similar to the actual material behavior with minor discrepancy. This discrepancy possibly originates from the differences in deformation moduli of different specimens. However, as the major concern of the proposed model is to simulate shear dilation, the observed discrepancy is acceptable.

The predictive capability of the proposed model is also evaluated in a triaxial extension mode. The reduced triaxial extension test $(R T E)$, which induces shearing by reducing the axial stress after hydrostatic loading stage, was performed in the laboratory and predicted by the proposed model. As shown in Fig. 11, the proposed model predicts the deformation, induced by hydrostatic stress unloading, similar to the actual ones.

Overall, the proposed model seems to be capable of reasonably predicting the deformational behavior of weak sandstones over a wide range of confining pressures and stress paths. 
Table 3

Corresponding parameters for the analyzed tunnel

\begin{tabular}{llll}
\hline Type of model & Parameter & Remark \\
\hline Proposed model & $a$ & Material parameter & Material parameter \\
& $b$ & Poisson's ratio & Material parameter \\
& $v$ & Slope of the yield criterion & 20 \\
& $e$ & Interception of the yield criterion & 0.21 \\
Elasto-plastic model (Drucker-Prager model) & $\alpha$ & Young's modulus & 0.16 \\
$82 \mathrm{kPa}$ & Poisson's ratio & Slope of failure criteria \\
& $k$ & Interception of failure criteria & 0.21 \\
& $E$ & &
\end{tabular}

${ }^{\mathrm{a}}$ Associated flow rule is assumed.

\section{Application to tunnel excavations}

As the proposed model for weak sandstones is capable of modeling material responses realistically with considerations of anisotropic softening, the impact of such behavior to the deformation induced by tunnel excavations can be accordingly analyzed through numerical analyses. The proposed constitutive model was incorporated into a finite element code $A B A Q U S$ and is then used to predict the wall deformation of the tunnel.

A well-known tunneling project in Taiwan is selected as a case study. This tunnel, with a height of $16 \mathrm{~m}$, a width of $12.4 \mathrm{~m}$, and a thickness of overburden ranging from 20 to $170 \mathrm{~m}$, was constructed in the area of the Western foothill of Taiwan, in which weak sedimentary rocks prevail. The rock mass along the tunnel was mostly the aforementioned Mushan sandstone. Drill and blast method was used to excavate this tunnel, and the excavation was done in two stages: top heading excavation and invert excavation.

Two constitutive models, linear-elastic-perfectly-plastic with Drucker-Prager yield criterion and the proposed model, are used to analyze the sidewall deformation of the studied tunnel, and the material parameters and properties are listed in Table 3 based on the studied rock mass. The deformational behaviors of the Drucker-Prager model, the proposed model, and the actual deformation obtained from tests are compared in Fig. 12. It can be seen that the Drucker-Prager model describes a linear volumetric contraction upon increasing hydrostatic pressures and no shear contraction or dilation before the weak rock fails.

A section of the tunnel, known to have suffered from ground squeezing, is shown in Fig. 13 based on the geometry described in Jeng et al. [1]. The tunnel was analyzed in three steps: geostatic condition with $K_{o}=1.0$, top heading excavation and invert excavation. The results of the analysis are presented subsequently.

Fig. 14 illustrates the variations of the shear modulus $G$ in the cross section throughout the analysis. It could be observed that significant degradations of the shear moduli $G$ at crown, sidewall, and invert take place during top heading excavation (stage 2 ). These moduli remain very

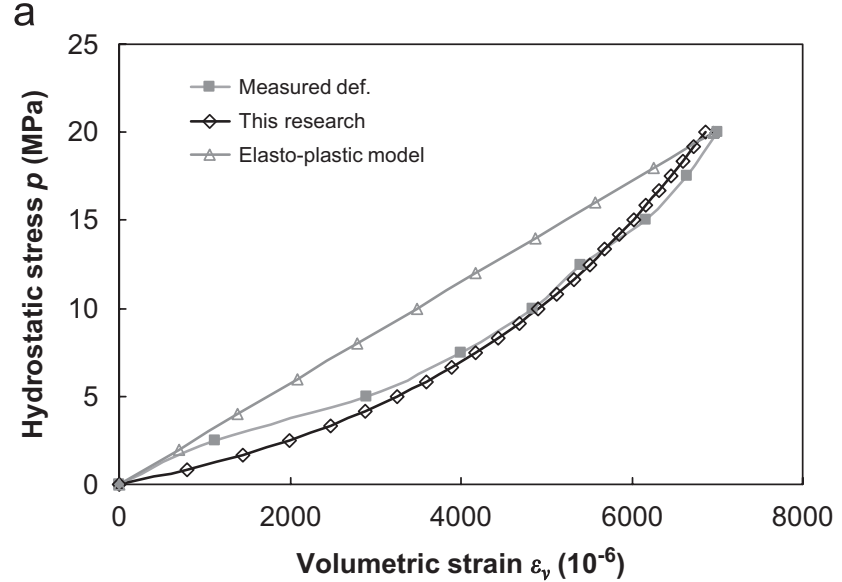

b

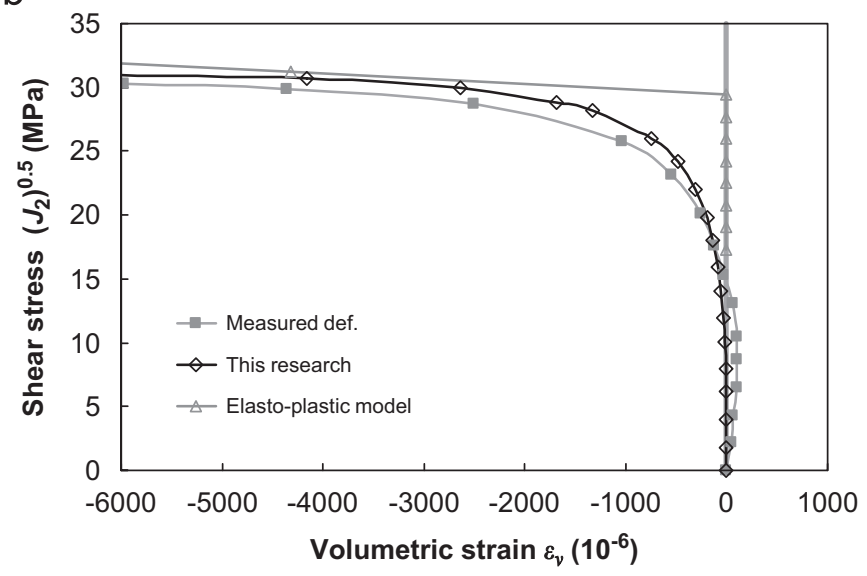

Fig. 12. Comparison of the deformational curves obtained by the Drucker-Prager model, the proposed model and the experiments.

low at the subsequent stage. It is found that softening initiates at the upper sidewalls first, then at the invert, and at last at the crown. Finally, the deformational modulus distribution, including Young's modulus and shear modulus, are shown in Fig. 15, which reveals that significant softening occurred all around the tunnel, and the tunnel invert has the most degradation. 


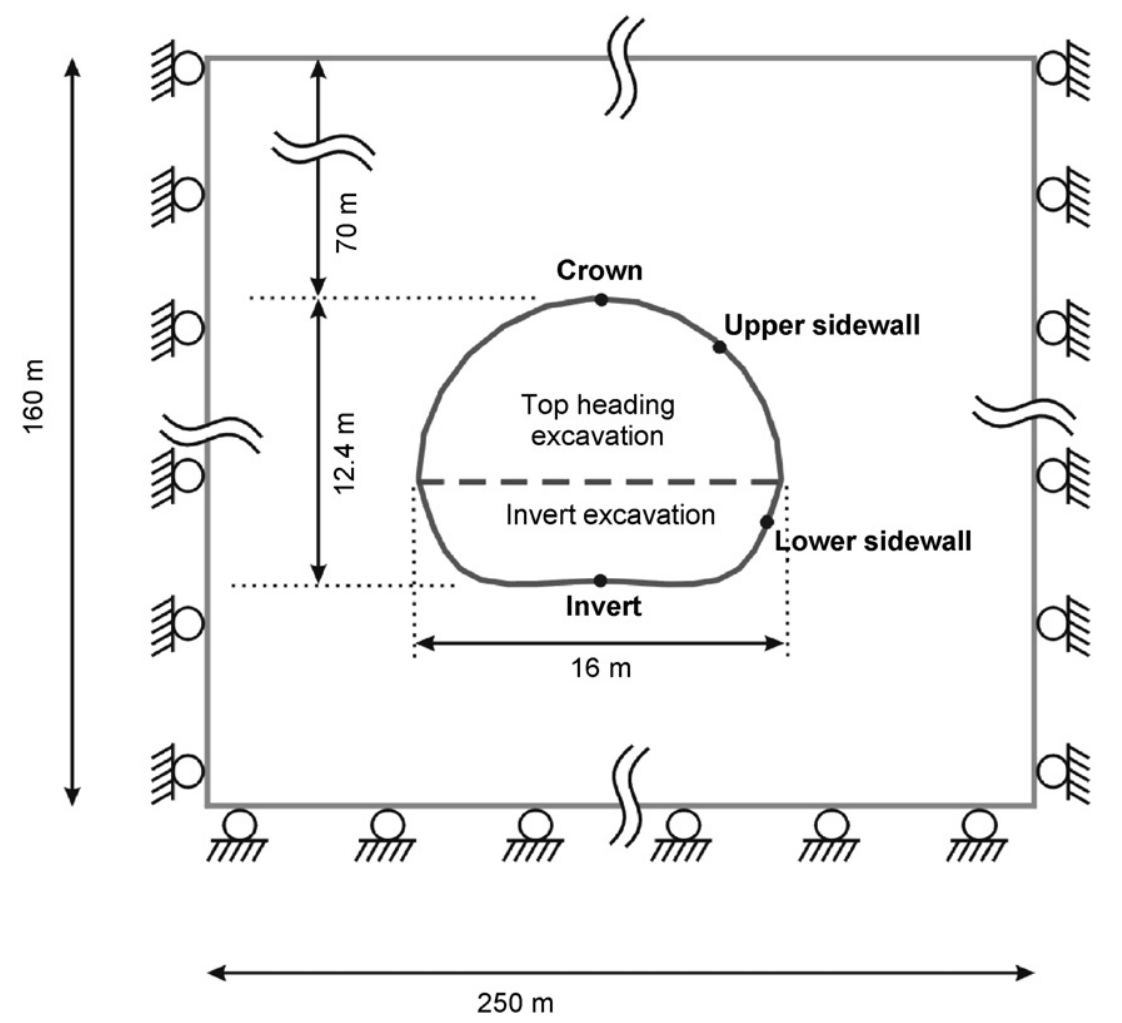

Fig. 13. Schematic illustration of the geometry of the studied tunnel.

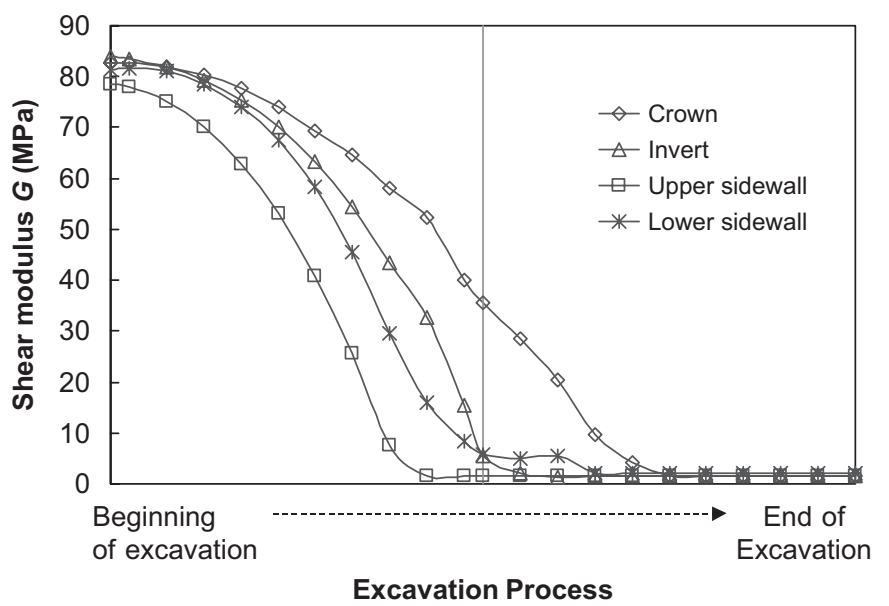

Fig. 14. Variations of the shear modulus $G$ around the cross section during analysis. The shear modulus $G$ at crown, sidewalls and invert initiate degradation at stage 2 (top heading). The tunnelwas excavated from the top heading to the invert, which was also followed by the numerical analysis. The abscissa represents the number of steps by which the elements within the tunnel were removed step by step.

The stress paths at the crown, the invert, and sidewalls are shown in Fig. 16. Similar stress paths close to RTE path are seen at the crown and at the invert; namely, the material is increasingly sheared while the hydrostatic pressure decreases. For sidewalls, however, the stress path is similar to CTC path. At last, the stresses at the crown, the invert, and the upper sidewall of inner section reach the failure envelope.

The inward displacements at selected locations on tunnel walls, calculated from finite element calculations based on the two material models used, are summarized in Table 4. It shows that the calculated inward displacements based on the proposed model are larger all over the tunnel section (including the crown, sidewalls and the invert) compared to those predicted by the elasto-plastic model. The actual crown settlement ranges from $14-30 \mathrm{~cm}$. As such, without incorporating plasticity, the proposed model has predicted a crown settlement closer to the reality than the one predicted by using the conventional elasto-plastic model.

What accounts for the significant amount of inward ground displacement movements obtained by the proposed model can be further explored by examining the volumetric strain distributions of the two models. Figs. 17(a) and (b) show the distribution of volumetric deformation around the tunnel obtained by the elasto-plastic model and the proposed model, respectively. As shown in Fig. 17(a), a dilation zone beneath the invert (the shaded areas) was developed based on the elasto-plastic model. Consequently, the elasto-plastic model predicts greater displacements at the invert than at other locations. However, the dilation zone calculated by the proposed model is much larger than that calculated by the elasto-plastic model, especially above the crown and beneath the invert, as shown in Fig. 17(b). As a result, more dilation pushes the crown further inward, as revealed by the proposed model. 
a

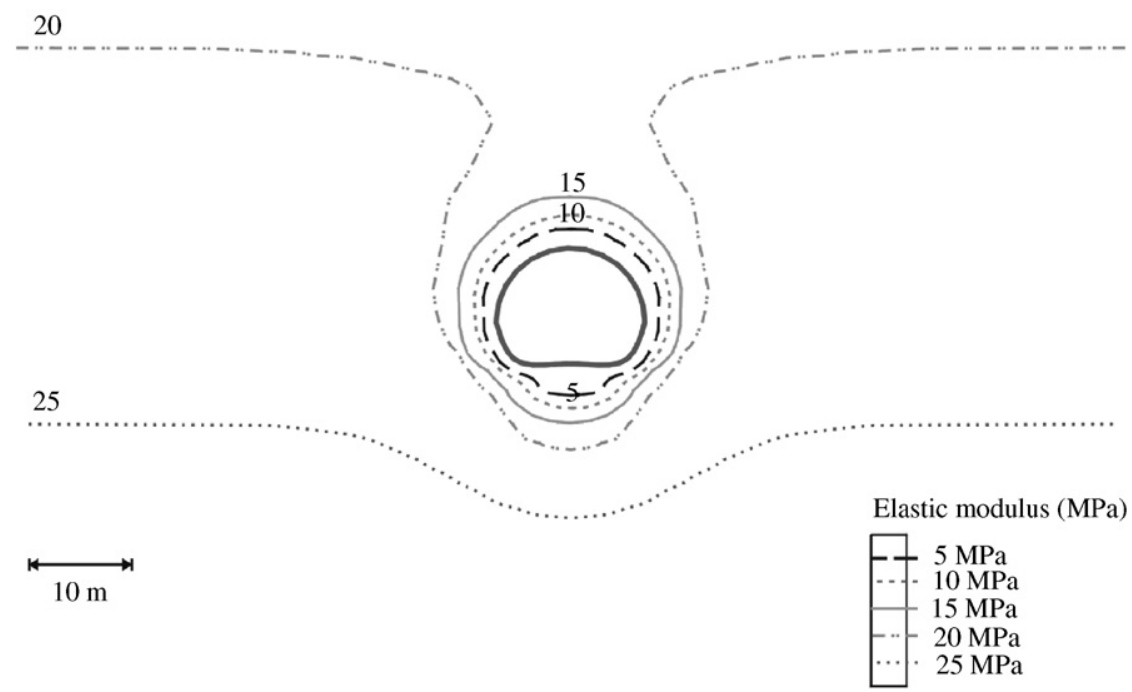

b

Elastic modulus E

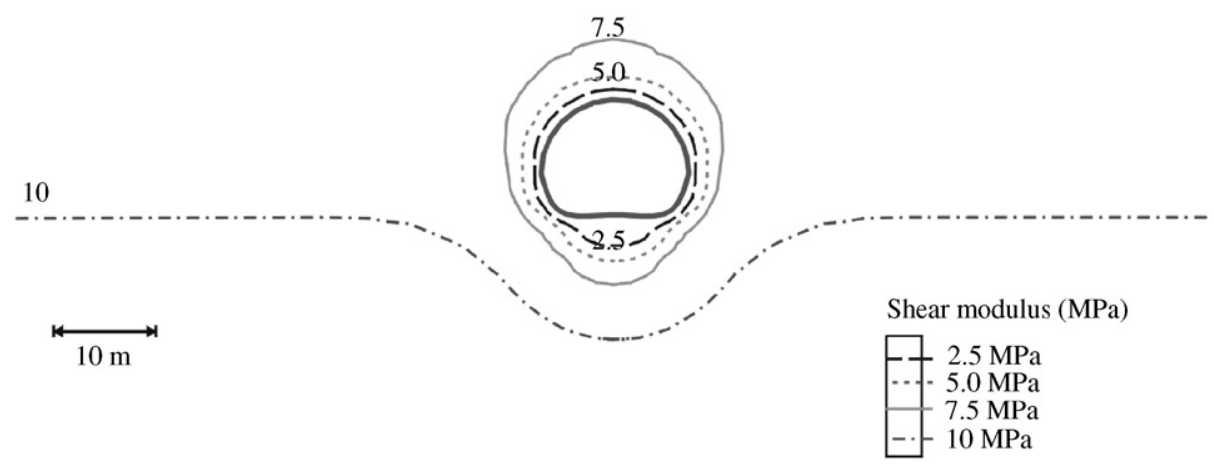

Shear modulus $\mathrm{G}$

Fig. 15. Distribution of $E$ and $G$ after tunnel excavation: (a) Young's modulus $E$; (b) shear modulus $G$.

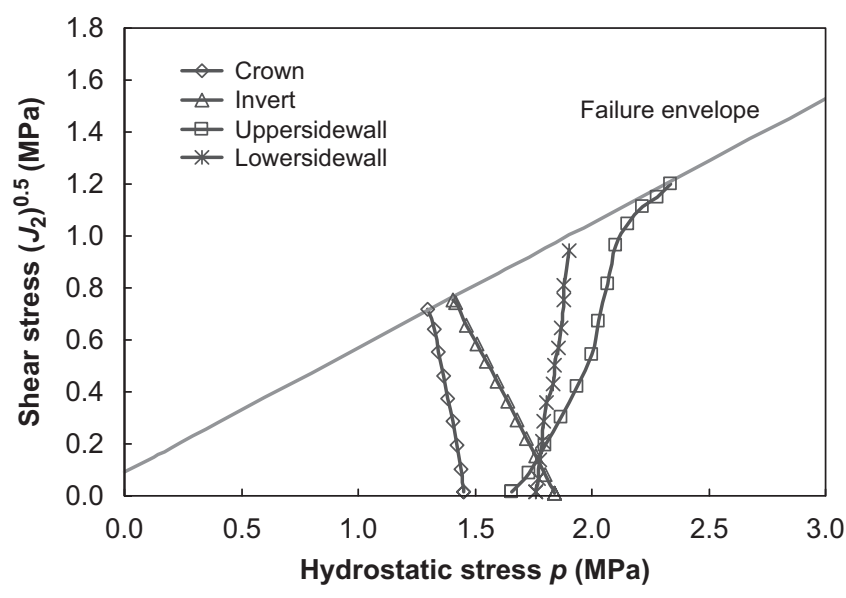

Fig. 16. Stress paths of the materials located at the crown, invert and sidewalls during tunnel excavation.
Table 4

Inward displacements predicted by two models

\begin{tabular}{lclc}
\hline Model/displacement $(\mathrm{cm})$ & Crown & Sidewall & Invert \\
\hline Proposed model & 10.2 & 8.6 & 15.8 \\
Elasto-plastic model & 6.3 & 6.5 & 12.6
\end{tabular}

The shear dilation prior to yielding accounts for the larger extent of the dilation zone, which cannot be simulated by the conventional elasto-plastic models since they would not allow dilation in the elastic range.

Remarkably, the crown settlement predicted by the proposed constitutive model is about $10.2 \mathrm{~cm}$, which is closer to, but still somewhat underestimated, the measured crown settlement of $14-30 \mathrm{~cm} \mathrm{[1].} \mathrm{Therefore,} \mathrm{other} \mathrm{factors}$ 

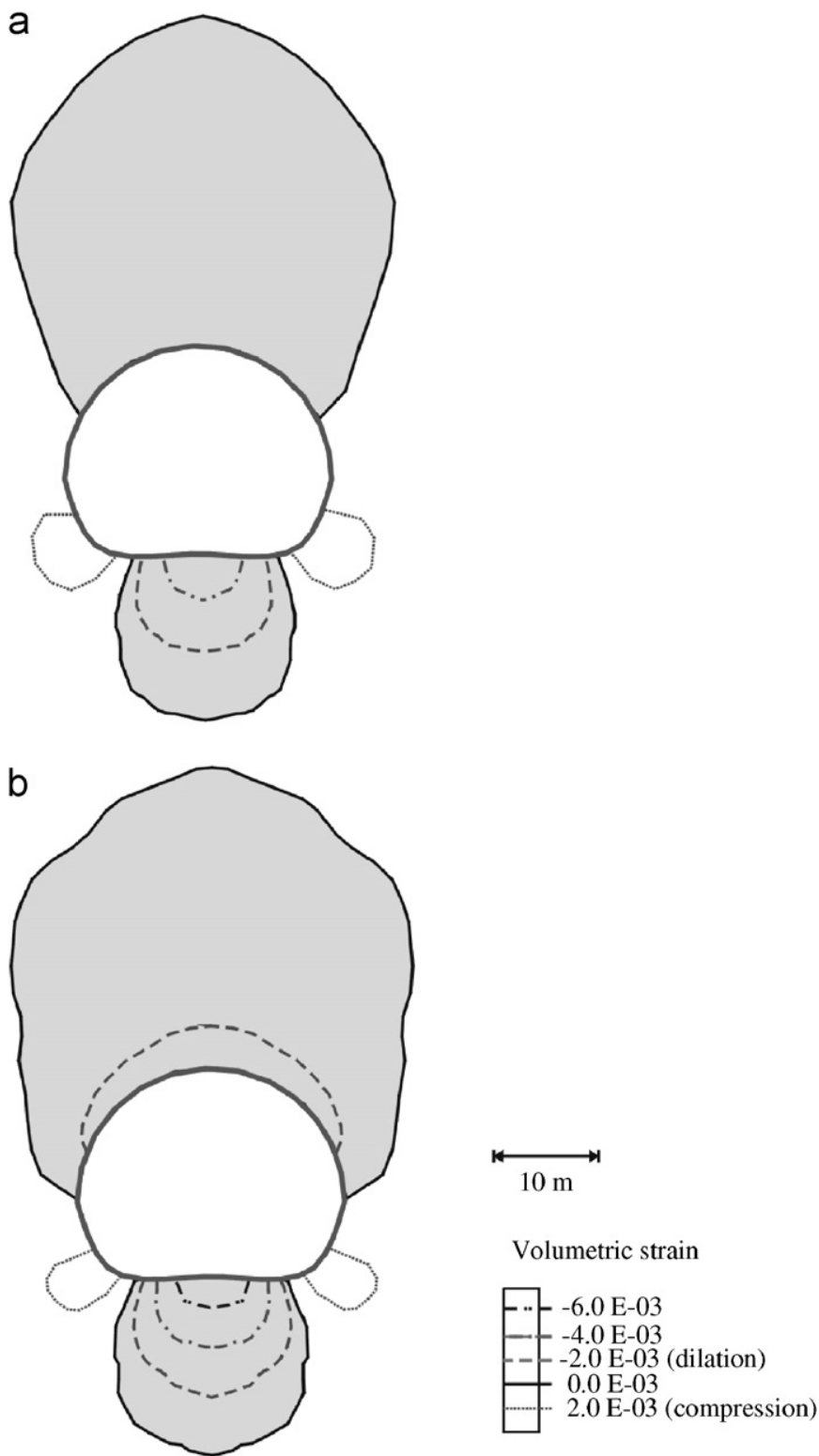

Fig. 17. Volumetric deformation predicted by different models. Dilation zones are illustrated by shaded areas. (a) Elasto-plastic model; (b) Proposed model.

such as scale effect and creep may account for the discrepancy and require further study.

\section{Conclusion}

An innovative constitutive model has been proposed for weak sandstones to describe the deformational characteristics exhibited by weak sandstones and other geomaterials. This proposed constitutive model is characterized by the following features: (1) the non-linear volumetric deformation under hydrostatic loading; (2) the significant shear dilation prior to the failure state; (3) modulus stiffening under hydrostatic loading and anisotropic softening under shearing condition; and (4) being versatile in various stress paths.

The stress-strain relationship of the model, which accounts for the variations of modulus $E$ and $G$ through different loading conditions, was formulated based on the framework of linear elasticity. Under hydrostatic stress condition, the weak sandstone is viewed as an isotropic material, and its stiffness increases as confining pressure arises. However, when the weak sandstone is subjected to shearing, the material not only becomes anisotropic but also softens. Such deformational characteristics have been observed through numerous experiments in the literature. As such, the modulus degradation is expressed as a decaying binomial function of the shear stress, and the shear modulus for the plane parallel to the maximum principal stress varies differently from those in the other two directions, accounting for shear-induced anisotropy. Finally, the proposed model was tested through comparisons between experimental data and model predictions and found to be versatile for various stress paths.

Compared to other sophisticated elasto-plastic models, the proposed model needs only six material parameters, all of which have physical meaning and can be obtained easily. The model has been incorporated into the finite element program $A B A Q U S$ and used to analyze a squeezing tunnel case in Taiwan. Comparing prediction results between using the proposed model and conventional elasto-plastic models, closer to reality and larger crown settlement owing to larger extent of dilation zones was observed. Overall, the proposed model can reasonably describe the deformation behavior, with significant shear dilation prior to the failure state for weak sandstones.

\section{Acknowledgments}

The research is supported by the National Science Council of Taiwan, Grant no. NSC-95-2221-E-002-263. The authors are grateful to the editor and two anonymous reviewers, whose advice has substantial improved the scientific soundness of this work.

\section{References}

[1] Jeng FS, Lin ML, Huang TH. Study of the geological barriers of the tunnels in Northern Taiwan (in Chinese). Taipei: Ministry of Transportation, research report, 1996.

[2] Jeng FS, Weng MC, Lin ML, Huang TH. Influence of petrographic parameters on geotechnical properties of Tertiary sandstones from Taiwan. Eng Geol 2004;73:71-91.

[3] Brown ET, editor. Rock characterization, testing, and monitoring ISRM suggested methods. New York: Pergamon; 1981.

[4] Weng MC. Mechanical characteristics and the relations with microstructure factors of foothill sandstones. $\mathrm{PhD}$ dissertation. Taipei: National Taiwan University Civil Engineering; 2002.

[5] Weng MC, Jeng FS, Huang TH, Lin ML. Characterizing the deformation behavior of tertiary sandstones. Int J Rock Mech Min Sci 2005;42:388-401.

[6] Zimmerman RW. Compressibility of Sandstones. Amsterdam: Elsevier; 1991. 
[7] Wu B, King MS, Hudson JA. Stress-induced ultrasonic wave velocity anisotropy in a sandstone. Int J Rock Mech Min Sci 1991;28:101-7.

[8] David C, Menendez B, Darot M. Influence of stress-induced and thermal cracking on physical properties and microstructure of $\mathrm{La}$ Peyratte granite. Int J Rock Mech Min Sci 1999;36:433-48.

[9] Sayers CM. Stress-induced ultrasonic wave velocity anisotropy in fractured rock. Ultrasonics 1988;26:311-7.

[10] Sayers CM, Van Munster JG, King MS. Stress-induced ultrasonic anisotropy in Berea sandstone. Int $\mathbf{J}$ Rock Mech Min Sci 1990;27:429-36.

[11] Sayers CM. Stress-dependent elastic anisotropy of sandstones. Geophys Prospect 2002;50:85-95.

[12] Ita SL, Cook NGW, Myer LR, Nihei KT. Effects of stress anisotropy on the static and dynamic properties of Berea sandstone. Int J Rock Mech Min Sci 1993;30:785-8.
[13] Lockner DA, Beeler NM. Stress-induced anisotropic poroelasticity response in sandstone. In: Proc. 16th ASCE eng mech conf, Seattle, 2003, pp. 16-18

[14] Olsson W. Development of anisotropy in the incremental shear moduli for rock undergoing inelastic deformation. Mech Mater 1995;21:231-42.

[15] Tatsuoka F, Shibuya S. Deformation characteristics of soils and rocks from field and laboratory tests. Rept Inst Indust Sci 1992;37:136.

[16] LoPresti DCF, Pallara O, Lancellotta R, Armandi M, Maniscalco R. Monotonic and cyclic loading behavior of two sands at small strains. ASTM Geotech Test J 1993;16:409-24.

[17] Tatsuoka F, Jardine RJ, LoPresti DCF, DiBenedetto H, Kodaka T. Characterizing the pre-failure deformation properties of geomaterials. In: 14th int conf soil mech found eng, vol 4. 1997. p 35. 